defence. The patient had solid defence in law - a) non-insane automatism and b) involuntary intoxication. Based on the evidential test alone, I fail to see why the CPS decided to prosecute the patient. It is submitted the CPS failed to apply the test properly or at all. The CPS has covered itself but with glory.

Can any lesson be learnt from this case? It is my tried and tested practice that all postoperative instructions (both verbal and written) are always given to the accompanying responsible person (but never to the patient), emphasising that the patient may not remember very much for the rest of the day and therefore [s] he should not, among other actions, sign anything important.

S. Appiah-Anane

Specialist Oral Surgeon, Barrister 1. Quick [1973] Q.B. 910 C.A.

DOI: 10.1038/sj.bdj.2012.423

\section{RESIGNED TO PENSION LOGIC}

Sir, as a female dentist, I am going to have to wait longer for my pensions than I originally expected and, with everyone else, will receive a lot less from my private pensions than originally predicted. This is not surprising as life expectancy has increased. In 1967 male life expectancy was 70.5 years, just over five years after the 65th birthday. In 2011 male life expectancy is 78, 13 years after the 65th birthday. We cannot expect the same percentage and number of years' pension contribution to fund our increased requirements.

Like many, I have medical conditions which prevent me from working full time, but I can work part-time and hopefully should be able to do so for a number of years to come. Pensions related to average earnings seem a sensible option, since more people may have to consider working part-time as we have to work longer.

Although not overjoyed with this prospect, I am resigned to its logic.

H. Butler, Dorset DOI: 10.1038/sj.bdj.2012.424

\section{MANAGING ASPIRATION}

Sir, a 68-year-old female presented to our A\&tE department with dyspnoea, a productive cough and intense chest pain on inspiration. She was diagnosed with a left lower lobe pneumonia and pleural empyema, caused by aspiration of dental amalgam, which had occurred some months earlier.

Whilst in A\&tE, the patient had a hypoxic cardiac arrest which required two cycles of CPR and emergency drugs, along with intubation and ventilation, before her circulation returned. She was admitted to the ICU for continued mechanical ventilation and antibiotic therapy. A CT scan of her chest was performed once the patient's condition had stabilised (Figs 1-2).

The dental amalgam was removed with rigid bronchoscopy, however, if this had failed, then a thoracotomy and pulmonary lobectomy would have been necessary. She eventually made a full recovery.

Foreign body aspiration is a well recognised complication of routine dental care. A wide variety of aspirated foreign bodies have been reported from teeth and restorative materials to instruments. ${ }^{3}$ The probability of aspiration is increased by the patient lying in a supine position for dental treatment.

Patient groups that are at increased risk of aspiration include the elderly, complete denture wearers, sedated patients, patients with organic brain disease such as Parkinson's, and those with neurological disabilities. ${ }^{2}$ These patients may have a decreased gag reflex, functional swallowing impairment or other abnormalities of the protective airway mechanism. ${ }^{1,2}$

Common early signs of foreign body inhalation include coughing, choking, inspiratory stridor, paradoxical breathing, decreased oxygen saturation and cyanosis. ${ }^{2}$ Early complications can lead to cardiac arrest and laryngeal oedema. Long term retention of a foreign body in the lung can lead to vocal cord paralysis, pneumonia, pneumothorax and death. ${ }^{2}$

The key to managing aspiration is of course prevention, ${ }^{1}$ but in the emergent situation, the patient should be quickly put into the reverse Trendelenburg position $^{2}$ and encouraged to cough. If coughing fails to relieve the obstruction, then back slaps and abdominal thrusts must be performed. If this is not rapidly successful then the patient must be transferred to hospital. An asympto-

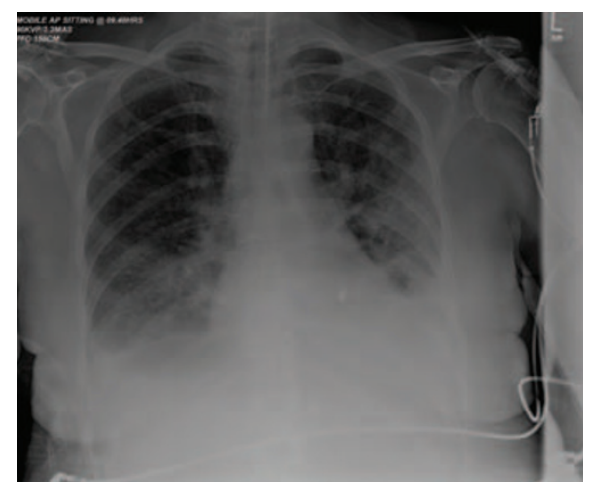

Fig. 1 Chest radiograph showing foreign body present in left lung

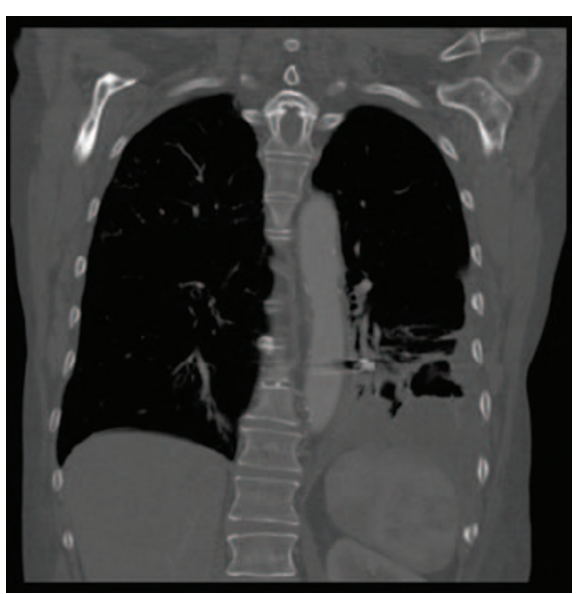

Fig. 2 CT scan showing a radiopaque foreign body obstructing the left lower lobe bronchus causing distal collapse and consolidation of the lung with an associated empyema

matic patient must still be referred on an urgent basis to hospital as it cannot be assumed that the object has been swallowed and not inhaled.

Bronchoscopy is the treatment of choice for removing inhaled foreign bodies. ${ }^{2}$ Cases where the foreign body persists for more than 24 hours have been associated with increased morbidity and a longer hospital stay. Intense mucosal inflammation and granulation tissue may then impede the removal of the foreign body. ${ }^{2}$

We would like to remind the dental practitioner of a few simple preventive measures which can help to decrease the risk of aspiration: identification of at-risk patients, ${ }^{2}$ the use of rubber dam for endodontic and restorative treatment, ${ }^{3}$ the use of a gauze partition at the back of the oral cavity, ${ }^{3}$ and the invaluable assistance of an attentive and effective dental nurse. ${ }^{1}$

R. Mann, B. Srinivasan, A. Baker Southampton 